\title{
Why Phenotypic Drug Susceptibility Testing of Mycobacterium Tuberculosis to First-Line Drugs is not Sufficient for Proper Management of Drug-Resistant and Multidrug-Resistant Tuberculosis?
}

\section{Suhail Ahmad}

Department of Microbiology, Faculty of Medicine, Health Sciences Center, Kuwait University, Kuwait

*Corresponding author: Suhail Ahmad, Department of Microbiology, Faculty of Medicine, Health Sciences Center, Kuwait University, Kuwait, Tel: 00965-24636503; Email: suhail_ah@hsc.edu.kw

Received date: January 18, 2018; Accepted date: January 22, 2018; Published date: January 30, 2018

Copyright: $@ 2018$ Ahmad S. This is an open-access article distributed under the terms of the Creative Commons Attribution License, which permits unrestricted use, distribution, and reproduction in any medium, provided the original author and source are credited.

\section{Editorial}

Tuberculosis (TB) is a major infectious disease of global proportions inflicting a heavy toll on human populations. Active TB disease is caused mainly by inhalation of droplet nuclei containing few bacilli exhaled by sputum smear-positive pulmonary TB patients (open TB) during close human contact [1]. Primary infection with Mycobacterium tuberculosis, however, leads to clinically active TB disease in only $\sim 10 \%$ of the exposed individuals. In the remaining subjects, an effective immune response mounted by the host arrests multiplication of tubercle bacilli, however, complete sterilization is achieved in only a sub-set of individuals [1]. In the remaining subjects, infection is only contained but not eradicated as some bacilli escape killing and persist in granulomatous lesions (latent TB infection). The latent infection may remain dormant for a long-time; however, $M$. tuberculosis can also resuscitate and cause active $\mathrm{TB}$, typically due to waning of the immune response [1]. Nearly one-third of the human population is latently infected with tubercle bacilli and 5\%-10\% of the infected individuals will eventually develop active TB disease during their life-time [1].

The global burden of TB and the associated morbidity and mortality are enormous. According to the latest annual survey conducted by the World Health Organization (WHO), there were an estimated 10.4 million active TB disease cases in 2016 [2]. The annual number of incident TB cases varied widely among different countries, from under 10 per 100000 populations in most high-income countries to $150-300$ per 100000 in most of the 30 high TB burden countries [2]. More than half of all active TB cases occurred in only five (China, India, Indonesia, the Phillippines and Pakistan) countries [2]. An estimated 1.7 million individuals died from TB in 2016, making TB as the ninth leading cause of death worldwide [2]. Most of the deaths were attributed to the resistance of $M$. tuberculosis to one or more anti-TB drugs.

The anti-TB drugs are categorized as first-line (most effective), second-line (less effective, more toxic) and third-line (agents of unproven efficacy) drugs based on efficacy and tolerability [3-5]. Firstline drugs (rifampicin, isoniazid, ethambutol, pyrazinamide and streptomycin) are highly efficacious, fairly affordable, relatively less toxic and mostly bactericidal oral agents suitable for combination therapy [3-5]. Streptomycin, an intramuscularly administered drug, is now mostly used as a second-line agent due to requirement for frequent patient's visits to health care facilities and higher rates of resistance among $M$. tuberculosis isolates [3-5]. Other rifamycins (rifabutin and rifapentine), though more expensive, may also be used in place of rifampicin in select patient populations [3-5].
Second-line agents are divided into three different groups; injectable aminoglycosides (kanamycin and amikacin) and cyclic polypeptides (capreomycin and viomycin), flouroquinolones (including ofloxacin, levofloxacin, moxifloxacin and gatifloxacin), and mainly bacteriostatic oral agents like ethionamide, prothionamide, D-cycloserine, terizidone, and para-amino salicylic acid [3-5]. Third-line reinforcing agents include linezolid, amoxicillin-clavulanate, meropenemclavulanate, clofazimine and thiacetazone. The second-line and thirdline agents are mainly used for the treatment of multidrug-resistant (MDR) (resistant at least to rifampicin and isoniazid, the two most effective first-line drugs) and extensively drug-resistant (XDR) (additionally resistant to a fluoroquinolone plus kanamycin/amikacin/ capreomycin) TB (MDR/XDR-TB) cases due to lower efficacy and serious side effects [3-6].

The widespread occurrence of drug-resistant (DR)-TB, MDR-TB and XDR-TB strains of $M$. tuberculosis is a serious threat to global TB control efforts [5,7]. In 2016, an estimated 600000 new TB cases were resistant to rifampicin of which 490000 cases were resistant to both, rifampicin and isoniazid (MDR-TB) [2]. It is also estimated that nearly $10 \%$ of all MDR-TB cases now have XDR-TB which is often fatal. Several countries, including India, Iran and South Africa, have also reported totally drug-resistant (TDR)-TB strains that are apparently resistant to all tested first-line, second-line and third-line anti-TB drugs $[7,8]$. However, this (TDR-TB) disease entity is currently not endorsed by WHO since drug susceptibility testing (DST) for many second-line/third-line drugs are poorly reproducible (ranging from $50 \%$ to $80 \%$ ), the number of drugs tested varies among reference laboratories and the existing category of XDR-TB already encompasses extensive drug resistance to most active anti-TB drugs [9].

Compared to fully drug-susceptible (pansusceptible)-TB, treatment of patients with DR-TB and MDR-TB is much more difficult due to lengthy (9-24 months), more expensive and more toxic drug regimens and the patients often experience clinical failure or disease relapse $[5,7,10]$. The WHO has further categorized infection with $M$. tuberculosis strains resistant only to rifampicin and isoniazid without additional resistance to other first-line drugs as uncomplicated MDRTB. Treatment of uncomplicated MDR-TB is easier and success rate for uncomplicated MDR-TB is higher compared to treatment of MDR-TB resistant to additional first-line drugs $[5,7,10]$. Globally, treatment success rates for TB, MDR-TB and XDR-TB have been recorded as $83 \%, 54 \%$, and $30 \%$, respectively [2]. Thirty-five countries in Asia and Africa have also introduced shorter (9-12 months) drug regimens for treatment of MDR-TB, with treatment success rates of nearly $90 \%$. Several $(>50)$ countries have also started using newer (bedaquiline and delamanid) drugs in treatment regimens for MDR/XDR-TB [2]. Unsuccessful treatment of MDR-TB is also a risk factor for XDR-TB, 
Citation: Ahmad S (2018) Why Phenotypic Drug Susceptibility Testing of Mycobacterium Tuberculosis to First-Line Drugs is not Sufficient for Proper Management of Drug-Resistant and Multidrug-Resistant Tuberculosis?. J Bacteriol Parasitol 9: E128. doi: $10.4172 / 2155-9597.1000 \mathrm{e} 128$

Page 2 of 4

which is very difficult to treat in most of the developing countries $[5,7,10]$.

Accurate DST of M. tuberculosis in clinical specimens and culture isolates to first-line drugs is crucial for the diagnosis of DR-TB and $\mathrm{MDR} / \mathrm{XDR}-\mathrm{TB}$ for proper patient management and to limit further transmission of MDR-TB and development of XDR-TB [5,11-15]. Phenotypic DST of $M$. tuberculosis by solid (Lowenstein-Jensen) medium-based proportion method is considered as the gold standard for first-line (except pyrazinamide) and important second-line (injectable agents such as kanamycin, amikacin and capreomycin and new generation fluoroquinoloes) drugs. However, the method is very slow as it requires 4-6 weeks to report results [16-18]. Commercial liquid culture systems and molecular assays have been developed and endorsed by WHO and Centers for Disease Control and Prevention (CDC) for more rapid detection of drug resistance in $M$. tuberculosis [15,18-20]. The liquid-broth-based semiautomated, radiometric BACTEC 460TB system accurately performed DST of $M$. tuberculosis for both, first-line (including pyrazinamide) and second-line drugs for more than two decades, reporting results within 10-14 days and was considered as an accurate and reliable alternative to the solid mediumbased method $[15,17,18]$.

The concerns for safe disposal of radioactivity have led to the development of fully automated culture systems such as Bactec Mycobacteria Growth Indicator Tube (MGIT) 960 system, MB/BacT system and Versa TREK system with similar turnaround time $[15,17,18]$. These rapid systems, particularly MGIT 960 system have now replaced BACTEC 460TB system in clinical microbiology laboratories around the world. Although the performance of MGIT 960 system has been excellet for two first-line drugs, isoniazid and streptomycin, and important second-line (new generation fluoroquinolones and injectable agents) drugs, recent studies have shown poor performance of MGIT 960 system for M. tuberculosis isolates carrying specific resistance conferring mutations in target genes for other first-line drugs [15,18,21-23].

Resistance of M. tuberculosis to rifampicin in $95-97 \%$ isolates is due to mutations in an 81-base pair (bp) rifampicin resistance determining region (RRDR) of $r p o B$ gene while the remaining 3-5\% isolates contain mutations in $\mathrm{N}$-terminal or cluster II region of the $r p o B$ gene or in other genes [24]. The MGIT 960 system fails to detect rifampicin resistance in $M$. tuberculosis strains exhibiting low-level (minimum inhibitory concentration, MIC of 0.5-2.0 $\mu \mathrm{g} / \mathrm{ml}$ ) resistance [25-27]. These low-level rifampicin-resistant strains with increased MICs below the critical concentration mostly contain mutations within RRDR or at codon 572 within cluster II region of the $r p o B$ gene [25-27]. Ironically, $\mathrm{I} 572 \mathrm{~F}$ mutation in cluster II region of the $r p o B$ gene was accurately detected by the (now discontinued) BACTEC 460TB system [28]. The disputed (generally missed by rapid phenotypic DST methods) mutations accounted for $>10 \%$ of all $r p o B$ mutations in $M$. tuberculosis strains from patients with failing therapy or experiencing relapse in Bangladesh and Democratic Republic of Congo. The clinical significance of some disputed mutations is suggested by gene replacement studies [29]. Furthermore, patients infected with $M$. tuberculosis strains with disputed $r p o B$ mutations often fail treatment or relapse just like patients infected with $M$. tuberculosis strains carrying canonical $r p o B$ mutations [30-32]. These findings call for adaptation of the standard DST by MGIT 960 system for greater accuracy of rifampicin resistance detection. The findings also suggest that a susceptible result should be confirmed by molecular testing when the suspicion for rifampicin resistance (such as previous history of anti-TB therapy, failing therapy, relapse or history of close contact with a patient with rifampicin-resistant/MDR-TB) is high.

Pyrazinamide is a key drug for first-line treatment of pansusceptible TB and second-line treatment regimens of DR-TB/MDR$\mathrm{TB}$ as the drug is active against persister bacilli in acidic environment (within macrophages) [33]. Phenotypic DST of M. tuberculosis for pyrazinamide (most effective at $\mathrm{pH}$ 5.6) is not routinely performed because of the requirements for precise acidic conditions which prevent the growth of about $20 \%$ of the isolates [34,35]. Furthermore, the inoculum size also has profound effects on DST results as larger inoculum may lead to alkalization of the medium causing false PZA resistance [34-36]. Nearly $90 \%$ of pyrazinamide-resistant $M$. tuberculosis isolates contain mutations in pncA gene [37]. Due to difficulties in accurate phenotypic pyrazinamide DST, WHO is currently considering pncA-based molecular diagnostics as the recommended approach for this purpose.

Ethambutol, a slow-acting first-line drug, interferes with $M$. tuberculosis growth by inhibition of one of three arabinosyltransferases (encoded by embCAB operon) that are required for the synthesis of arabinogalactan, a component of the mycobacterial cell wall [38]. Mutations in embCAB operon lead to ethambutol resistance but only modestly (3-8 fold) increase its MIC while highlevel resistance develops later due to acquisition of additional mutations either in embCAB operon or in other genes $[29,39,40]$. Mutations in $e m b B$ gene are more common and mostly occur at codons 306, 406 and 497 [41-43]. Phenotypic DST methods for ethambutol often report false susceptibility of $M$. tuberculosis. The radiometric BACTEC 460TB system (now discontinued) was more accurate compared to the current MGIT 960 system, particularly for isolates containing embB mutations [29,39-43]. Recent studies have shown that patients infected with embB mutants should be considered as having ethambutol-resistant TB even if the isolates appear to be ethambutol-susceptible by phenotypic DST methods to avoid evolution of secondary mutations and selection of fully drug-resistant strains $[22,39,40]$. False susceptibility to ethambutol is not critical for the treatment of drug-susceptible TB, however, it is detrimental for successful treatment of MDR-TB as drug regimens for this disease entity should not include ineffective first-line drugs $[5,7,10,44]$.

Compared to the slow and/or inaccurate DST of M. tuberculosis by culture-based methods, molecular methods rapidly (within 1-2 days) detect genetic mutations associated with drug resistance and mainly include hybridization-based assays, PCR-sequencing of select panel of target genes and whole genome sequencing of $M$. tuberculosis in clinical specimens and culture isolates $[19,20,28,45,46]$.

Hybridization-based assays include GeneXpert MTB/RIF assay for the diagnosis of active TB disease and its resistance to rifampicin $[2,19,47]$. GenoType MTBDRplus line probe assay detects resistance to first-line drugs, rifampicin and isoniazid (MDR-TB) while GenoType MTBDRsl detects resistance of MDR-TB strains for second-line drugs, fluoroquinoloes and injectable aminoglycoside/cyclic peptide drugs for detection of XDR-TB [48-50]. DNA microarrays also detect resistance to various combinations of first-line and/or second-line drugs with sensitivity of $\sim 90 \%$ for detection of MDR/XDR-TB [51,52]. A disadvantage of these methods is the rare possibility of false resistance detection due to silent (synonymous) mutations in target regions $[20,27]$.

PCR-sequencing has been used for detecting resistance to one or several first-line and second-line drugs and to confirm the results of 
Citation: Ahmad S (2018) Why Phenotypic Drug Susceptibility Testing of Mycobacterium Tuberculosis to First-Line Drugs is not Sufficient for Proper Management of Drug-Resistant and Multidrug-Resistant Tuberculosis?. J Bacteriol Parasitol 9: E128. doi: $10.4172 / 2155-9597.1000 \mathrm{e} 128$

Page 3 of 4

resistance detection by other methods $[22,26-28,43,45]$. The sensitivity of PCR-sequencing varies considerably according to the number and regions of drug resistance-associated loci included for each drug and the frequency of specific mutations in these loci at different geographical locations/ethnic groups of TB patients [42,43,48,53-55]. However, this approach is time consuming and technically demanding and is being rapidly replaced by whole genome sequencing [14,46,48].

Whole-genome sequencing (WGS) is an attractive alternative to characterise common and rare mutations in $M$. tuberculosis strains predicting resistance for all first-line, second-line, third-line and new drugs and to guide appropriate drug regimens for DR-TB and MDR/ XDR-TB [46,56-58]. The method has also been used directly on patient samples for same day diagnosis [59]. Some developed countries have already started to use whole genome sequencing routinely for the diagnosis of $\mathrm{TB}$, detection of drug resistance and typing of $M$. tuberculosis for epidemiological purposes [56-59]. However, the high cost of equipment and reagents and the requirement of technical expertise and bioinformatic support make this method difficult to implement, at present, in resource-poor developing countries for proper patient management where DR-TB and MDR/XDR-TB are endemic.

\section{Acknowledgements}

The research work from Prof. Ahmad's research group was supported by Kuwait University Research Sector grant MI03/16.

\section{References}

1. Ahmad S (2010) New approaches in the diagnosis and treatment of latent tuberculosis infection. Respir Res 11: 169.

2. World Health Organization (2017) Global tuberculosis report 2017. Geneva, Switzerland.

3. Blumberg HM, Burman WJ, Chaisson RE, Daley CL, Etkind SC, et al (2003) American Thoracic Society/Centers for Disease Control and Prevention/Infectious Diseases Society of America: treatment of tuberculosis. Am J Respir Crit Care Med 167: 603-662.

4. Ahmad S, Mokaddas E (2009) Recent advances in the diagnosis and treatment of multidrug-resistant tuberculosis. Resp Med 103: 1777-1790.

5. Ahmad S, Mokaddas E (2014) Current status and future trends in the diagnosis and treatment of drug-susceptible and multidrug-resistant tuberculosis. J Infect Pub Health 7: 75-91.

6. Dooley KE, Obuku EA, Durakovic N, Belitsky V, Mitnick C, et al. (2013) World Health Organization group 5 drugs for the treatment of drugresistant tuberculosis: unclear efficacy or untapped potential? J Infect Dis 207: 1352-1358.

7. Dheda K, Gumbo T, Maartens G, Dooley KE, McNerney R, et al. (2017) The epidemiology, pathogenesis, transmission, diagnosis, and management of multidrug-resistant, extensively drug-resistant, and incurable tuberculosis. Lancet Respir Med 5: 291-360.

8. Dheda K, Gumbo T, Gandhi NR, Murray M, Theron G, et al. (2014) Global control of tuberculosis: from extensively drug-resistant to untreatable tuberculosis. Lancet Respir Med 2: 321-338.

9. Cegielski P, Nunn P, Kurbatova EV, Weyer K, Dalton TL, et al. (2012) Challenges and controversies in defining totally drug-resistant tuberculosis. Emerg Infect Dis 18: e2.

10. Dheda K, Chang KC, Guglielmetti L, Furin J, Schaaf HS, et al. (2017) Clinical management of adults and children with multidrug-resistant and extensively drug-resistant tuberculosis. Clin Microbiol Infect 23: 131-140.

11. Drobniewski F, Nikolayevskyy V, Balabanova Y, Bang D, Papaventsis D (2012) Diagnosis of tuberculosis and drug resistance: what can new tools bring us? Int J Tuberc Lung Dis 16: 860-870.
12. World Health Organization (2014) Drug-resistant TB, surveillance and response: supplement to global tuberculosis report 2014. Geneva, Switzerland.

13. Matteelli A, Centis R, D'Ambrosio L, Sotgiu G, Tadolini M, et al. (2016) WHO strategies for the programmatic management of drug-resistant tuberculosis. Expert Rev Respir Med 10: 991-1002.

14. Sulis G, Centis R, Sotgiu G, D'Ambrosio L, Pontali E, et al. (2016) Recent developments in the diagnosis and management of tuberculosis. NPJ Prim Care Respir Med 26: 16078.

15. Schön T, Miotto P, Köser CU, Viveiros M, Böttger E, et al. (2017) Mycobacterium tuberculosis drug-resistance testing: challenges, recent developments and perspectives. Clin Microbiol Infect 23: 154-160.

16. World Health Organization (1998) Guidelines for surveillance of drug resistance in tuberculosis. WHO and the International Union Against Tuberculosis and Lung Disease. Int J Tuberc Lung Dis 2: 72-89.

17. Piersimoni C, Olivieri A, Benacchio L, Scarparo C (2006) Current perspectives on drug susceptibility testing of Mycobacterium tuberculosis complex: the automated nonradiometric systems. J Clin Microbiol 44: 20-28.

18. Palomino JC, Martin A, Von Groll A, Portaels F (2008) Rapid culturebased methods for drug-resistance detection in Mycobacterium tuberculosis. J Microbiol Methods 75: 161-166.

19. World Health Organization (2013) Automated real-time nucleic acid amplification technology for rapid and simultaneous detection of tuberculosis and rifampicin resistance: Xpert MTB/RIF assay for the diagnosis of pulmonary and extrapulmonary TB in adults and children. Policy Update. Geneva, Switzerland.

20. Nathavitharana RR, Cudahy PG, Schumacher SG, Steingart KR, Pai M, et al. (2017) Accuracy of line probe assays for the diagnosis of pulmonary and multidrug-resistant tuberculosis: a systematic review and metaanalysis. Eur Respir J 49: 1601075.

21. Angra PK, Taylor TH, Iademarco MF, Metchock B, Astles JR, et al. (2012) Performance of tuberculosis drug susceptibility testing in U.S. laboratories from 1994 to 2008. J Clin Microbiol 50: 1233-1239.

22. Ahmad S, Mokaddas E, Al-Mutairi N, Eldeen HS, Mohammadi S (2016) Discordance across phenotypic and molecular methods for drug susceptibility testing of drug-resistant Mycobacterium tuberculosis isolates in a low TB incidence country. PLoS One 11: e0153563.

23. Maningi NE, Malinga LA, Antiabong JF, Lekalakala RM, Mbelle NM (2017) Comparison of line probe assay to BACTEC MGIT 960 system for susceptibility testing of first and second-line anti-tuberculosis drugs in a referral laboratory in South Africa. BMC Infect Dis 17: 795.

24. Telenti A, Imboden P, Marchesi F, Lowrie D, Cole S, et al. (1993) Detection of rifampicin-resistance mutations in Mycobacterium tuberculosis. Lancet 341: 647-650.

25. Rigouts L, Gumusboga M, de Rijk WB, Nduwamahoro E, Uwizeye C, et al. (2013) Rifampin resistance missed in automated liquid culture system for Mycobacterium tuberculosis isolates with specific rpoB mutations. J Clin Microbiol 51: 2641-2645.

26. Van Deun A, Aung KJ, Bola V, Lebeke R, Hossain MA, et al. (2013) Rifampin drug resistance tests for tuberculosis: challenging the gold standard. J Clin Microbiol 51: 2633-2640.

27. Mokaddas E, Ahmad S, Eldeen HS, Al-Mutairi N (2015) Discordance between Xpert MTB/RIF assay and Bactec MGIT 960 culture system for detection of rifampin-resistant Mycobacterium tuberculosis isolates in a country with a low tuberculosis (TB) incidence. J Clin Microbiol 53: 1351-1354.

28. Al-Mutairi N, Ahmad S, Mokaddas E (2011) Performance comparison of four methods for rapid detection of multidrug-resistant Mycobacterium tuberculosis strains. Int J Tuberc Lung Dis 15: 110-115.

29. Nebenzahl-Guimaraes H, Jacobson KR, Farhat MR, Murray MB (2014) Systematic review of allelic exchange experiments aimed at identifying mutations that confer drug resistance in Mycobacterium tuberculosis. J Antimicrob Chemother 69: 331-342. 
Citation: Ahmad S (2018) Why Phenotypic Drug Susceptibility Testing of Mycobacterium Tuberculosis to First-Line Drugs is not Sufficient for Proper Management of Drug-Resistant and Multidrug-Resistant Tuberculosis?. J Bacteriol Parasitol 9: E128. doi: $10.4172 / 2155-9597.1000 \mathrm{e} 128$

Page 4 of 4

30. Van Ingen J, Aarnoutse R, de Vries G, Boeree MJ, van Soolingen D (2011) Low-level rifampicin-resistant Mycobacterium tuberculosis strains raise a new therapeutic challenge. Int J Tuberc Lung Dis 15: 990-992.

31. Van Deun A, Aung KJM, Hossain MA, de Rijk P, Gumusboga M, et al. (2015) Disputed rpoB mutations can frequently cause important rifampicin resistance among new tuberculosis patients. Int J Tuberc Lung Dis 19: 185-190.

32. Shah NS, Grace Lin SY, Barry PM, Cheng YN, Schecter G, et al. (2016) Clinical impact on tuberculosis treatment outcomes of discordance between molecular and growth-based assays for rifampin resistance, California 2003-2013. Open Forum Infect Dis 3: 150.

33. Mitchison DA (2000) Role of individual drugs in the chemotherapy of tuberculosis. Int J Tuberc Lung Dis 4: 796-806.

34. Zhang Y, Permar S, Sun Z (2002) Conditions that may affect the results of susceptibility of Mycobacterium tuberculosis to pyrazinamide. J Med Microbiol 51: 42-49.

35. Zhang Y, Chang KC, Leung CC, Yew WW, Gicquel B, et al. (2012) 'ZSMDR-TB' versus 'ZR-MDR-TB': improving treatment of MDR-TB by identifying pyrazinamide susceptibility. Emerg Microbes Infect 1: e5.

36. Morlock GP, Tyrrell FC, Baynham D, Escuyer VE, Green N, et al. (2017) Using reduced inoculum densities of Mycobacterium tuberculosis in MGIT pyrazinamide susceptibility testing to prevent false-resistant results and improve accuracy: a multicenter evaluation. Tuberc Res Treat 2017: 3748163.

37. Ramirez-Busby SM, Valafar F (2015) Systematic review of mutations in pyrazinamidase associated with pyrazinamide resistance in Mycobacterium tuberculosis clinical isolates. Antimicrob Agents Chemother 59: 5267-5277.

38. Telenti A, Philipp W, Sreevatsan S, Bernasconi C, Stockbauer KE, et al. (1997) The emb operon, a unique gene cluster of Mycobacterium tuberculosis involved in resistance to ethambutol. Nat Med 3: 567-570.

39. Safi H, Sayers B, Hazbón MH, Alland D (2008) Transfer of embB codon 306 mutations into clinical Mycobacterium tuberculosis strains alters susceptibility to ethambutol, isoniazid, and rifampin. Antimicrob Agents Chemother 52: 2027-2034.

40. Lingaraju S, Rigouts L, Gupta A, Lee J, Umubyeyi AN, et al. (2016) Geographic differences in the contribution of ubiA mutations to highlevel ethambutol resistance in Mycobacterium tuberculosis. Antimicrob Agents Chemother 60: 4101-4105.

41. Ahmad S, Jaber AA, Mokaddas E (2007) Frequency of embB codon 306 mutations in ethambutol-susceptible and -resistant clinical Mycobacterium tuberculosis isolates in Kuwait. Tuberculosis 87: 123-129.

42. Cheng S, Cui Z, Li Y, Hu Z (2014) Diagnostic accuracy of a molecular drug susceptibility testing method for the antituberculosis drug ethambutol: a systematic review and meta-analysis. J Clin Microbiol 52: 2913-2924.

43. Al-Mutairi NM, Ahmad S, Mokaddas E (2018) Molecular screening versus phenotypic susceptibility testing of multidrug-resistant Mycobacterium tuberculosis isolates for streptomycin and ethambutol. Microb Drug Resist.

44. Ahuja SD, Ashkin D, Avendano M, Banerjee R, Bauer M, et al. (2012) Multidrug resistant pulmonary tuberculosis treatment regimens and patient outcomes: an individual patient data meta-analysis of 9,153 patients. PLoS Med 9: e1001300.

45. Campbell PJ, Morlock GP, Sikes RD, Dalton TL, Metchock B, et al. (2011) Molecular detection of mutations associated with first- and second-line drug resistance compared with conventional drug susceptibility testing of Mycobacterium tuberculosis. Antimicrob Agents Chemother 55: 2032-2041.

46. Köser CU, Bryant JM, Becq J, Török ME, Ellington MJ, et al. (2013) Whole-genome sequencing for rapid susceptibility testing of $M$. tuberculosis. N Engl J Med 369: 290-292.

47. Steingart KR, Schiller I, Horne DJ, Pai M, Boehme CC, et al. (2014) Xpert $^{\oplus}$ MTB/RIF assay for pulmonary tuberculosis and rifampicin resistance in adults. Cochrane Database Syst Rev 1: CD009593.

48. Drobniewski F, Cooke M, Jordan J, Casali N, Mugwagwa T, et al. (2015) Systematic review, meta-analysis and economic modelling of molecular diagnostic tests for antibiotic resistance in tuberculosis. Health Technol Assess 19: 1-188.

49. Bai Y, Wang Y, Shao C, Hao Y, Jin Y (2016) GenoType MTBDRplus assay for rapid detection of multidrug resistance in Mycobacterium tuberculosis: a meta-analysis. PLoS One 11: e0150321.

50. Mao X, Ke Z, Shi X, Liu S, Tang B, et al. (2015) Diagnosis of drug resistance to fluoroquinolones, amikacin, capreomycin, kanamycin and ethambutol with Genotype MTBDRsl assay: a meta-analysis. Ann Clin Lab Sci 45: 533-544.

51. Linger Y, Kukhtin A, Golova J, Perov A, Lambarqui A, et al. (2014) Simplified microarray system for simultaneously detecting rifampin, isoniazid, ethambutol, and streptomycin resistance markers in Mycobacterium tuberculosis. J Clin Microbiol 52: 2100-2107.

52. Zhu L, Liu Q, Martinez L, Shi J, Chen C, et al. (2015) Diagnostic value of GeneChip for detection of resistant Mycobacterium tuberculosis in patients with differing treatment histories. J Clin Microbiol 53: 131-135.

53. Abal AT, Ahmad S, Mokaddas E (2002) Variations in the occurrence of the S315T mutation within the katG gene in isoniazid-resistant clinical Mycobacterium tuberculosis isolates from Kuwait. Microb Drug Resist 8 99-105.

54. Yuan X, Zhang T, Kawakami K, Zhu J, Li H, et al. (2012) Molecular characterization of multidrug- and extensively drug-resistant Mycobacterium tuberculosis strains in Jiangxi, China. J Clin Microbiol 50: 2404-2413.

55. Ahmad S, Al-Mutairi NM, Mokaddas E (2012) Variations in the occurrence of specific rpoB mutations in rifampicin-resistant Mycobacterium tuberculosis isolates from patients of different ethnic groups in Kuwait. Indian J Med Res 135: 756-762.

56. Zhang H, Li D, Zhao L, Fleming J, Lin N, et al. (2013) Genome sequencing of 161 Mycobacterium tuberculosis isolates from China identifies genes and intergenic regions associated with drug resistance. Nat Genet 45: 1255-1260.

57. Witney AA, Gould KA, Arnold A, Coleman D, Delgado R, et al. (2015) Clinical application of whole-genome sequencing to inform treatment for multidrug-resistant tuberculosis cases. J Clin Microbiol 53: 1473-1483.

58. Arnold A, Witney AA, Vergnano S, Roche A, Cosgrove CA, et al. (2016) XDR-TB transmission in London: case management and contact tracing investigation assisted by early whole genome sequencing. J Infect 73: 210-218.

59. Votintseva AA, Bradley P, Pankhurst L, Del Ojo Elias C, Loose M, et al. (2017) Same-day diagnostic and surveillance data for tuberculosis via whole-genome sequencing of direct respiratory samples. J Clin Microbiol 55: 1285-1298. 